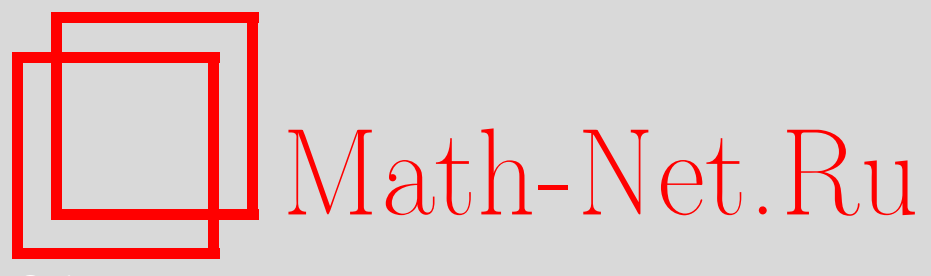

Т. А. Майоло, Ф. Делла Сала, Л. Мартина, Д. Солиани, Зацепление электронов во взаимодействующих молекулах, ТМФ, 2007, том 152, номер 2, 321-338

DOI: https://doi.org/10.4213/tmf6090

Использование Общероссийского математического портала Math-Net.Ru подразумевает, что вы прочитали и согласны с пользовательским соглашением http://www . mathnet.ru/rus/agreement

Параметры загрузки:

IP : 3.85 .5 .30

26 апреля 2023 г., 13:15:48

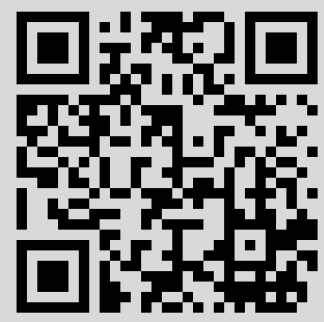




\title{
ЗАЦЕПЛЕНИЕ ЭЛЕКТРОНОВ ВО ВЗАИМОДЕЙСТВУЮЩИХ МОЛЕКУЛАХ
}

\begin{abstract}
Концепция квантового зацепления используется для придания физического значения энергии корреляции электронов в системах с взаимодействующими электронами. Электронные корреляции не могут наблюдаться непосредственно, они определяются как разность между точной энергией основного состояния многоэлектронного уравнения Шредингера и энергией Хартри-Фока. Корреляционная энергия вычисляется с помощью метода конфигурационных взаимодействий для молекулы водорода, и полученный результат сравнивается с зацеплением как функцией от расстояния между ядрами. В том же духе производится анализ димера этилена, который представляет собой простейшее органическое соединение, путем изменения взаимной ориентации и расстояния между молекулами, что позволяет найти конфигурацию, отвечающую максимальному зацеплению.
\end{abstract}

Ключевые слова: зацепление, энергия корреляции электронов, взаимодействующие молекулы.

\section{1. ВВЕДЕНИЕ}

Квантовое зацепление представляет собой термин, обычно отражающий существование в квантовых системах определенных корреляций, которые не допускают классической интерпретации. Оно оказывается полезным для увеличения взаимной информации, передаваемой по каналам с памятью, или для убыстрения таких квантовых процессов, как, например, факторизация по алгоритму Шора. Более того, зацепление представляет собой физическую величину, непосредственно измеряемую в терминах фон неймановской энтропии системы.

В последнее время значительные усилия были направлены на исследование квантового зацепления в связи с его возможными применениями в теории квантовой информации [1]. В самом деле, квантовое зацепление играет фундаментальную

${ }^{*}$ Dipartimento di Fisica dell'Università di Lecce, INFN, Sezione di Lecce, 73100 Lecce, Italy. E-mail: tina.maiolo@le.infn.it, Martina@le.infn.it, Soliani@le.infn.it

${ }^{\dagger}$ National Nanotechnology Laboratory; Consiglio Nazionale delle Ricerche - Instituto Nazionale per la Fisica della Materia di Lecce, 73100 Lecce, Italy. E-mail: fabio.dellasala@unile.it 
роль в квантовой телепортации [2], суперплотном кодировании [3], распределении квантовых ключей [4], квантовой криптографии [5]. Оно совершенно необходимо для экспоненциального ускорения процесса квантовых вычислений по сравнению с классическими вычислениями.

По этой причине немало усилий было направлено на описание характера этого явления, а экспериментальные измерения показали, что зацепление может влиять и на макроскопические свойства твердых тел [6]. Например, Чен с соавторами проанализировали зацепление основных состояний в XXZ-цепочке и в димеризованной гейзенберговской цепочке, равно как и на решетке типа лестницы-стремянки, использовав спин-спиновое взаимодействие и энтропию зацепления между выбранной подрешеткой спинов и оставшейся подсистемой [7]. Хе с соавторами нашли в работе [8] спектр энергий, парные корреляционные функции и степень зацепления двуэлектронных состояний в самоконцентрирующихся молекулах квантовой точки в сплаве InAs/GaAs, учитывая конфигурацию, в которой связанные состояния взаимодействуют между собой, и сравнили величины, найденные при различных приближениях. Бускеми с соавторами произвели численные расчеты динамики зацепления в процессах рассеяния двух неразличимых электронов, взаимодействующих посредством кулоновского потенциала в одно- и двумерных полупроводниковых наноструктурах [9]. Авторы при этом следовали идее Шлимана [10], развитой другими авторами [11], [12], согласно которой матрицу плотности в представлении пространственных координат можно использовать для вычисления энтропии фон Неймана. Этот подход основан на аналогии с разложением Шмидта для векторов состояний двух фермионных частиц: с помощью унитарного преобразования антисимметричная волновая функция может быть разложена по базису детерминантов Слейтера с минимальным числом неисчезающих членов. На основании величины, известной как слейтеровский ранг, можно построить критерий, позволяющий заключить, зацеплена система или нет. Этот критерий включает в себя вычисление энтропии фон Неймана для одночастично приведенной матрицы плотности. Для того чтобы применить зацепление в квантовых вычислениях, были исследованы и более сложные молекулы. Например, исследователи в Институте Хана-Мейтнер работают над реализацией молекулярных квантовых компьютеров, квантовые биты которых реализованы фуллеренами, со включенными отдельными атомами азота или фосфоpa [13].

Все это показывает важность исследования свойств некоторых молекул и межмолекулярных взаимодействий. Эти исследования являются предметом данной работы. Отправной точкой служит так называемая гипотеза Коллина [14], утверждающая, что энергия корреляций в молекулярных системах пропорциональна их энтропии. Эта гипотеза была подтверждена многочисленными экспериментами (см. работу Рамиреса с соавторами [15]) и принята Хуангом и Каисом, которые интерпретировали в работе [12] степень зацепления как величину энергии корреляции. На самом деле зацепление можно непосредственно измерить в терминах фон неймановской энтропии системы. Этот подход используется для придания физического смысла энергии корреляции электронов, которая не может быть непосредственно 
измерена, так как она определяется как разность между полной энергией данной молекулярной системы и энергией, полученной в рамках приближения Хартри-Фока. Метод Хартри-Фока обычно используется при решении уравнения Шредингера в многоэлектронном атоме или молекуле, описанной в приближении стационарных ядер (приближение Борна-Оппенгеймера) с помощью гамильтониана молекулярных электронов. Вычисление ошибки, связанной с таким приближением, в теории систем многих тел представляет собой серьезную трудность, решению которой посвящена обширная литература, см., например, книгу [16].

В данной работе представлено измерение зацепления электронов на примере двух различных двудольных систем, молекула $\mathrm{H}_{2}$ и димера этилена, в которых каждый атом водорода или каждая молекула этилена может рассматриваться как кубит. Мы сначала определяем, какой метод лучше применять для измерения зацепления, сравнивая фон неймановскую энтропию редуцированной матрицы плотности $S\left(\rho_{1}\right)$ с фон неймановской энтропией матрицы плотности $S(\rho)$ на примере молекулы $\mathrm{H}_{2}$. Заключив, что наиболее удобным методом оказывается второй, использующий энтропию $S(\rho)$, мы производим измерения зацепления в системе многих тел, представленной димером этилена, и сравниваем полученный результат с энергией электронных корреляций в системе. Наконец, используя неравенство Клейна для двудольной системы, мы получаем величину зацепления для взаимодействующих электронов, с помощью которой можно исследовать степень зацепления двух молекул, входящих в димер.

Статья организована следующим образом. В разделе 2 кратко описано, что такое зацепление [17] (т.е. определено, когда состояние отделимо или зацеплено [18]), и объяснено, почему мы выбираем фон неймановскую энтропию для оценки степени зацепления. В разделе 3 сначала проанализирован димер этилена, рассматриваемый как многоэлектронная система, вычислена его энергия корреляции, а затем произведено сравнение этой энергии с зацеплением как функцией от расстояния между двумя молекулами. В разделе 4 введена новая величина - зацепление взаимодействующих электронов, которая определяется как $S_{\mathrm{int}}=S\left[2 \mathrm{C}_{2} \mathrm{H}_{4}\right]-2 S\left[\mathrm{C}_{2} \mathrm{H}_{4}\right]$, для того чтобы найти зацепление между двумя молекулами димера. Затем с помощью вариации относительной ориентации и расстояния между молекулами определяется конфигурация, отвечающая максимальному зацеплению. В этом случае систему можно рассматривать как двудольную, а каждую молекулу - как кубит, если нас интересуют приложения к квантовым вычислениям. В разделе 5 объяснены некоторые детали вычислений, описан используемый пакет программ и показано, как подготавливаются входные данные для исследуемых составных систем. Наконец, раздел 6 содержит результаты и некоторые комментарии.

\section{2. ИЗМЕРЕНИЕ ЗАЦЕПЛЕНИЯ}

В квантовой механике, как правило, разграничиваются чистые и смешанные состояния в зависимости от информации о реальном состоянии, в котором находится физическая система [19]. 
Чистые состояния обычно вводятся в том случае, когда описывается физическая система, достоверно находящаяся в данном состоянии. Это состояние задается вектором $|\psi\rangle$ с единичной нормой в гильбертовом пространстве, связанном с системой. Эквивалентным образом чистое состояние можно задать оператором плотности $\rho=|\psi\rangle\langle\psi|$ таким, что $\operatorname{Tr} \rho=\operatorname{Tr} \rho^{2}=1$.

Если не известно точно, в каком состоянии находится система, и известна лишь вероятность $p_{i}$, с которой система будет находиться в чистом состоянии, заданном вектором $\left|\psi_{i}\right\rangle$, то обычно говорят, что система находится в смешанном состоянии, представленном оператором плотности $\rho=\sum_{i} p_{i}\left|\psi_{i}\right\rangle\left\langle\psi_{i}\right|=\sum_{i} p_{i} \rho_{i}$, где $p_{i} \geqslant 0$, $\sum_{i} p_{i}=1$, и $\rho_{i}-$ оператор плотности, связанный с чистым состоянием $\left|\psi_{i}\right\rangle$. В противоположность чистому состоянию смешанное состояние таково, что $\operatorname{Tr} \rho=1 \neq \operatorname{Tr} \rho^{2}$.

Наиболее важные следствия математической структуры пространства состояний проявляются при исследовании составных систем. В самом деле, рассмотрим физическую систему $T$, состоящую из двух различимых подсистем $A$ и $B$, состояния которых задаются элементами соответствующих гильбертовых пространств $\mathcal{H}_{A}$ и $\mathcal{H}_{B}$. Системе $T$ при этом соответствует $\mathcal{H}_{T}=\mathcal{H}_{A} \otimes \mathcal{H}_{B}$, а чистое состояние системы $T$ можно задать с помощью единичного вектора $|\psi\rangle \in \mathcal{H}_{T}$. Пусть $\left\{\left|\phi_{i}(A)\right\rangle\right\}_{i \in \mathbb{N}}$ и $\left\{\left|\chi_{j}(B)\right\rangle\right\}_{j \in \mathbb{N}}-$ ортонормированные базисы в соответствующих гильбертовых пространствах $\mathcal{H}_{A}$ и $\mathcal{H}_{B}$. Чистое состояние в $\mathcal{H}_{T}=\mathcal{H}_{A} \otimes \mathcal{H}_{B}$ можно при этом представить в виде $|\psi\rangle=\sum_{i, j} c_{i j}\left|\phi_{i}(A)\right\rangle \otimes\left|\chi_{j}(B)\right\rangle$, где $\sum_{i, j}\left|c_{i j}\right|^{2}=1$. Таким образом, если состояние $|\psi\rangle \in \mathcal{H}_{T}$ можно представить в виде тензорного произведения элемента пространства $\mathcal{H}_{A}$ и элемента пространства $\mathcal{H}_{B},|\psi\rangle=|\phi(A)\rangle \otimes|\chi(B)\rangle \equiv|\phi(A), \chi(B)\rangle$, то будем говорить, что $|\psi\rangle$ представляет собой чистое состояние, заданное в виде произведения (отделимое или факторизованное чистое состояние). Чистое состояние пары квантовых систем называется зацепленным, если оно не факторизуется. Согласно известной теореме фон Неймана [20], вектор $|\psi\rangle \in \mathcal{H}_{T}$ можно представить в виде так называемого шмидтовского разложения:

$$
|\psi\rangle=\sum_{i \geqslant 1} \sqrt{p_{i}}\left|\nu_{i}(A)\right\rangle\left|\lambda_{i}(B)\right\rangle .
$$

Тогда $|\psi\rangle$ представляет собой состояние в виде произведения, только если ровно один из шмидтовских коэффициентов отличен от нуля (если $i=1$ в сумме). Напротив, если ненулевыми оказываются более одного из шмидтовских коэффициентов (если $i>1$ в сумме), то чистое состояние оказывается зацепленным.

Рассмотрим в качестве простой двудольной системы молекулу $\mathrm{H}_{2}$, гильбертово пространство которой можно представить в виде

$$
\mathcal{H}=\left[\mathcal{L}^{m}(1) \otimes \mathcal{S}^{2}(1)\right] \otimes\left[\mathcal{L}^{m}(2) \otimes \mathcal{S}^{2}(2)\right]=\mathcal{C}^{2 m}(1) \otimes \mathcal{C}^{2 m}(2),
$$

где $\mathcal{L}$ и $\mathcal{S}$ задают соответствующие пространства орбиталей и спина; в выражении в скобках отмечено, к какому из двух электронов относятся эти пространства, а верхний индекс задает размерность пространства.

В представлении чисел заполнения $\left(n_{1} \uparrow, n_{1} \downarrow, n_{2} \uparrow, n_{2} \downarrow, \ldots, n_{m} \uparrow, n_{m} \downarrow\right)$ [21] нижние индексы указывают пространственное орбитальное число, и в каждом из 
пространств $\mathcal{C}^{m}$ введен ортонормированный базис

$$
\left\{\begin{array}{c}
\left|n_{1} \uparrow\right\rangle \\
\left|n_{1} \downarrow\right\rangle \\
\left|n_{2} \uparrow\right\rangle \\
\left|n_{2} \downarrow\right\rangle \\
\vdots \\
\left|n_{m} \uparrow\right\rangle \\
\left|n_{m} \downarrow\right\rangle
\end{array}\right\} \otimes\left\{\begin{array}{c}
\left|n_{1} \uparrow\right\rangle \\
\left|n_{1} \downarrow\right\rangle \\
\left|n_{2} \uparrow\right\rangle \\
\left|n_{2} \downarrow\right\rangle \\
\vdots \\
\left|n_{m} \uparrow\right\rangle \\
\left|n_{m} \downarrow\right\rangle
\end{array}\right\} .
$$

Чистое двухэлектронное состояние $|\Psi\rangle$ в этом представлении имеет вид

$$
|\Psi\rangle=\sum_{a=1}^{2 m} \sum_{b=1}^{2 m} \omega_{a, b} c_{a}^{\dagger} c_{b}^{\dagger}|0\rangle
$$

где $|0\rangle$ - вакуумное состояние, а коэффициенты $\omega_{a, b}$ удовлетворяют в соответствии с принципом исключения Паули соотношениям

$$
\omega_{a, b}=-\omega_{b, a}, \quad \omega_{i, i}=0,
$$

где $c^{\dagger}$ и $c$ - соответственно операторы рождения и уничтожения одночастичных состояний, действующие на вакуумное состояние следующим образом:

$$
\begin{aligned}
& 1 \equiv\left|n_{1} \uparrow\right\rangle, \quad 2 \equiv\left|n_{1} \downarrow\right\rangle, \\
& c_{a}^{\dagger} c_{b}^{\dagger}|0\rangle=|a b\rangle, \quad 3 \equiv\left|n_{2} \uparrow\right\rangle, \quad 4 \equiv\left|n_{2} \downarrow\right\rangle, \\
& a, b \in\{1,2,3,4, \ldots, 2 m\} \text {, } \\
& 2 m-1 \equiv\left|n_{m} \uparrow\right\rangle, \quad 2 m \equiv\left|n_{m} \downarrow\right\rangle .
\end{aligned}
$$

Коэффициенты $\omega_{a, b}$ можно вычислить с помощью метода конфигурационных взаимодействий [22], [23]. В частности, для молекулы $\mathrm{H}_{2}$ используется метод конфигурационных взаимодействий типа один-два (configuration interaction single-double (CISD) method), в котором учитываются только одинарные и парные возмущения:

$$
\left|\Psi^{\mathrm{CISD}}\right\rangle=c_{0}\left|\psi_{0}\right\rangle+\sum_{a r} c_{a}^{r}\left|\psi_{a}^{r}\right\rangle+\sum_{a<b, r<s} c_{a, b}^{r, s}\left|\psi_{a, b}^{r, s}\right\rangle,
$$

где $\left|\psi_{0}\right\rangle$ - волновая функция Хартри-Фока основного состояния, $c_{a}^{r}$ - коэффициент единичного возмущения из орбитали $a$ в орбиталь $r$, а $c_{a, b}^{r, s}-$ коэффициент парного возмущения из орбиталей $a$ и $b$ в орбитали $r$ и $s$. В представлении чисел заполнения волновая функция в CISD-методе задается выражением

$$
|\Psi\rangle=c_{0}\left|1_{1} 1_{2} 0_{3} 0_{4} \ldots\right\rangle+c_{1}^{3}\left|0_{1} 1_{2} 1_{3} 0_{4} \ldots\right\rangle+c_{2}^{4}\left|1_{1} 0_{2} 0_{3} 1_{4} \ldots\right\rangle+c_{1,2}^{3,4}\left|0_{1} 0_{2} 1_{3} 1_{4} \ldots\right\rangle+\cdots,
$$

где 1 и 0 отвечают присутствию и отсутствию электрона, соответственно, а нижний индекс задает номер узла, в котором находится электрон [24].

Чтобы решить, является состояние составной системы зацепленным или отделимым, необходимо построить соответствующую редуцированную матрицу плотности. 
В самом деле, вектор $|\psi\rangle$ задает состояние в виде произведения для составной системы тогда и только тогда, когда соответствующие операторы редуцированных плотностей задают чистые состояния (равнозначно, $|\psi\rangle$ - зацепленное состояние тогда и только тогда, когда соответствующие операторы редуцированных плотностей формально задают смешанные состояния). Таким образом, если необходимо определить степень зацепления, то следует вычислить степень смешанности для соответствующих редуцированных операторов плотностей (или матриц плотностей) [25]. Это вытекает из того обстоятельства, что зацепленное состояние порождает корреляции, информация о которых теряется, если наблюдению доступны только подсистемы, поскольку при этом мы фактически имеем смешанное состояние.

При описании степени случайности состояния и смешанности операторов плотности особенно важна энтропия фон Неймана, определяемая как

$$
S(\rho)=-\operatorname{Tr}\left(\rho \log _{2} \rho\right)
$$

Важно отметить, что $\rho$ представляет собой оператор с неотрицательным следом, в то время как $S$ не обязана быть неотрицательной. По аналогии с классической энтропией $S$ является количественной характеристикой случайности для состояния $\rho$. Более точно, энтропия $S$ равна нулю тогда и только тогда, когда состояние является чистым, и достигает максимума при $S(\rho)=\log _{2} d$, где $d$ - размерность гильбертова пространства. Другими словами, чем более смешанным оказывается оператор редуцированной плотности, тем более зацепленным становится исходное состояние, и этот результат можно рассматривать как основание для использования энтропии в качестве меры квантового зацепления. Тем самым, зацепление чистого состояния пары квантовых систем можно получить, рассмотрев энтропию любой системы, входящей в пару. Для вычисления степени электронного зацепления в молекуле $\mathrm{H}_{2}$, таким образом, необходимо вычислить фон неймановскую энтропию матрицы плотности, редуцированной по состояниям одного из электронов. Отталкиваясь от матрицы плотности $\rho=\left|\Psi^{\mathrm{CISD}}\right\rangle\left\langle\Psi^{\mathrm{CISD}}\right|$, можно получить редуцированную матрицу плотности, вычисляя частичный след по всем числам заполнения, кроме $n_{1} \uparrow$ (см. приложение):

$$
\begin{aligned}
\rho_{1}^{\mathrm{CISD}} & =\operatorname{Tr}_{\substack{1<i \leqslant m \\
1 \leqslant j \leqslant m}} \rho^{\mathrm{CISD}}= \\
& =\sum_{\substack{n_{i} \uparrow=0,1 \\
n_{j} \downarrow=0,1}}\left\langle n_{1} \downarrow, n_{2} \uparrow, n_{2} \downarrow, \ldots, n_{m} \uparrow, n_{m} \downarrow|\rho| n_{1} \downarrow, n_{2} \uparrow, n_{2} \downarrow, \ldots, n_{m} \uparrow, n_{m} \downarrow\right\rangle, \\
\rho_{1}^{\mathrm{CISD}} & =\left(\begin{array}{cc}
\sum_{i=1}^{m-1}\left|c_{1}^{2 i+1}\right|^{2}+\sum_{i, j=1}^{m-1}\left|c_{1,2}^{2 i+1,2 j+2}\right|^{2} & 0 \\
0 & \left|c_{0}\right|^{2}+\sum_{i=1}^{m-1}\left|c_{2}^{2 i+2}\right|^{2}
\end{array}\right) .
\end{aligned}
$$


Фон неймановская энтропия редуцированной матрицы плотности $\rho_{1}^{\text {CISD }}$ представляет собой степень зацепления:

$$
\begin{aligned}
S\left(\rho_{1}^{\mathrm{CISD}}\right)=- & \operatorname{Tr}\left(\rho_{1}^{\mathrm{CISD}} \log _{2} \rho_{1}^{\mathrm{CISD}}\right)= \\
=- & \left(\sum_{i}^{m-1}\left|c_{1}^{2 i+1}\right|^{2}+\sum_{i=1}^{m-1}\left|c_{1,2}^{2 i+1,2 i+2}\right|^{2}\right) \times \\
& \times \log _{2}\left(\sum_{i}^{m-1}\left|c_{1}^{2 i+1}\right|^{2}+\sum_{i=1}^{m-1}\left|c_{1,2}^{2 i+1,2 i+2}\right|^{2}\right)- \\
& -\left(\left|c_{0}\right|^{2}+\sum_{i=1}^{m-1}\left|c_{2}^{2 i+2}\right|^{2}\right) \log _{2}\left(\left|c_{0}\right|^{2}+\sum_{i=1}^{m-1}\left|c_{2}^{2 i+2}\right|^{2}\right) .
\end{aligned}
$$

Для того чтобы обобщить это метод на многочастичные системы, мы определяем степень зацепления как фон неймановскую энтропию матрицы плотности, отвечающей составной системе:

$$
S(\rho)=-\operatorname{Tr}\left(\rho \log _{2} \rho\right)=-\frac{1}{2}\left(\sum \alpha_{i} \log _{2} \alpha_{i}+\sum \beta_{i} \log _{2} \beta_{i}\right),
$$

где $\alpha_{i}$ и $\beta_{i}$ - так называемые естественные спиновые орбитали (собственные значения $\rho$ ). Мы сравним поведение зацепления как функции от межъядерного расстояния $R$, найденное с помощью CISD-метода расчета коэффициентов, с результатами метода естественных спиновых орбиталей.

На рис. 1 показано, что зацепление как фон неймановская энтропия редуцированной матрицы плотности $S\left(\rho_{1}^{\mathrm{CISD}}\right)$ обладает поведением, весьма похожим на то, которое получается, если рассчитывать зацепление как фон неймановскую энтропию матрицы плотности $S(\rho)$ составной системы. В частности, при малых межъядерных расстояниях две кривые, представляющие энтропии, перекрываются, а вне области связи приводят к различным результатам, хотя и имеют схожее поведение. Так как нас интересует качественное описание зацепления и его максимальное значение, можно пренебречь указанными расхождениями и при исследовании более сложных систем выбрать метод естественных спиновых орбиталей как основной.

\section{3. ЗАЦЕПЛЕНИЕ КАК МЕРА КОРРЕЛЯЦИИ ЭЛЕКТРОНОВ}

Покажем теперь, что зацепление можно рассматривать в качестве меры корреляции электронов [26]-[28]. Зацепление представляет собой физическую наблюдаемую, которую можно непосредственно измерить как фон неймановскую энтропию системы. Напротив, энергия корреляции электронов не может наблюдаться непосредственно, поскольку она определяется как разность между точной полной энергией данной молекулярной системы и энергией, полученной в соответствии с методом Хартри-Фока, где все межъядерные взаимодействия рассматриваются в приближении среднего поля:

$$
E_{\text {corr }}=E_{\mathrm{HF}}-E_{\text {exact }} .
$$




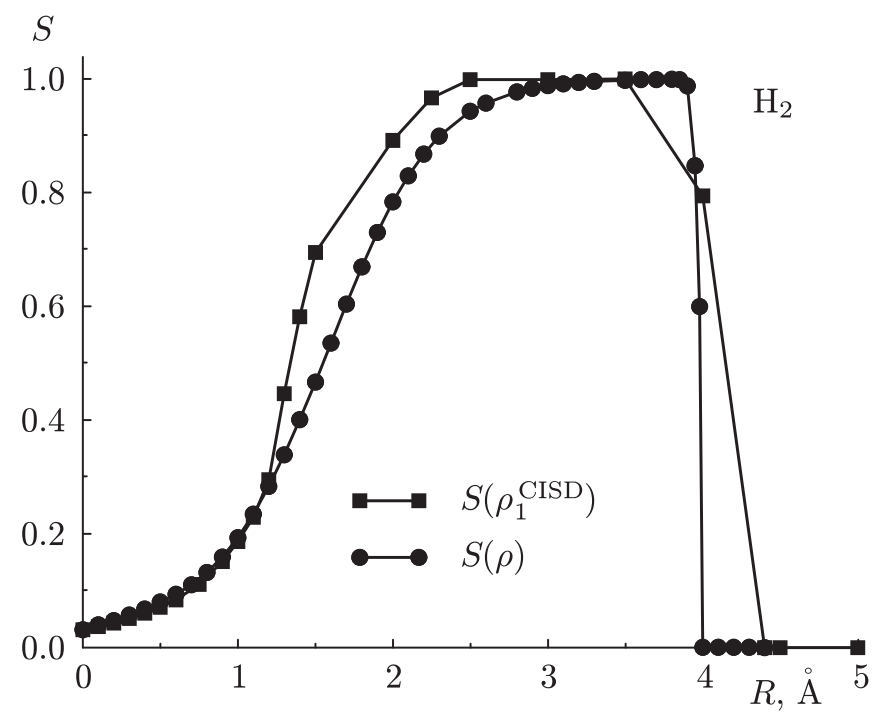

Рис. 1. Степень зацепления $S$ в зависимости от межъядерного расстояния, рассчитанная как энтропия $S\left(\rho_{1}^{\mathrm{CISD}}\right)$ матрицы плотности, редуцированной по состояниям одного электрона, и как энтропия $S(\rho)$ матрицы плотности составной системы.

Энергия корреляции есть энергия, которая проявляется, если позволить электронам совершенно не взаимодействовать друг с другом, а в методе Хартри-Фока необоснованно полагается, что межэлектронное отталкивание следует усреднять. Другими словами, точная волновая функция системы многих взаимодействующих электронов никогда не представляет собой один единственный определитель или простую комбинацию нескольких определителей. Эта ошибка возникает как раз за счет корреляций, которые оказываются аналогом квантового зацепления в отделимых системах и играют существенную роль при считывании квантовой информации в неотделимых системах.

В работе [12] авторы обсуждают образование молекулы $\mathrm{H}_{2}$ и демонстрируют качественное согласие между зацеплением и энергией корреляции как функцией расстояния между ядрами. Мы обобщим их аргументы на более сложные молекулы типа димера этилена. Фиксируя расстояние между плоскостями молекул, можно вычислить энергию корреляций электронов в димере с помощью процедуры, описанной в разделе 5. Степень зацепления для этой конфигурации можно рассчитать с помощью метода естественных спиновых орбиталей. Мы производим вычисления, изменяя расстояние между плоскостями или производя вращение при фиксированном расстоянии. Результаты расчета представлены на рис. 2а и 2б, соответствено.

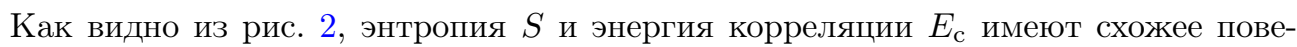
дение, хотя мы и использовали два различных масштаба при представлении этих величин. Если рассмотреть $S$ и $E_{\text {с }}$ как функции от расстояния между плоскостя- 


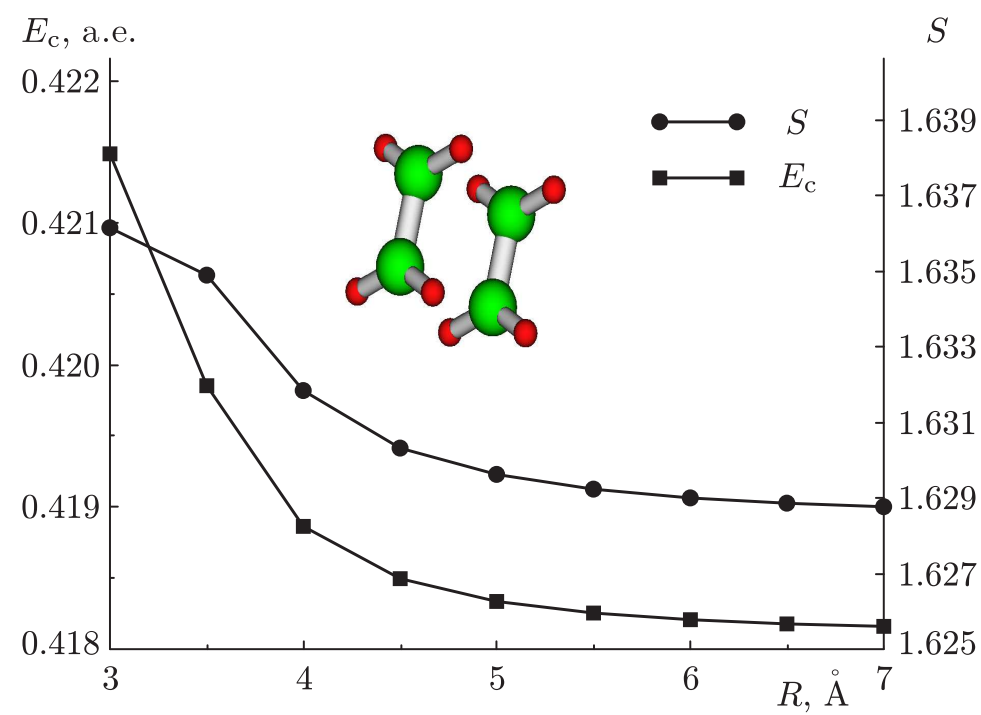

$a$

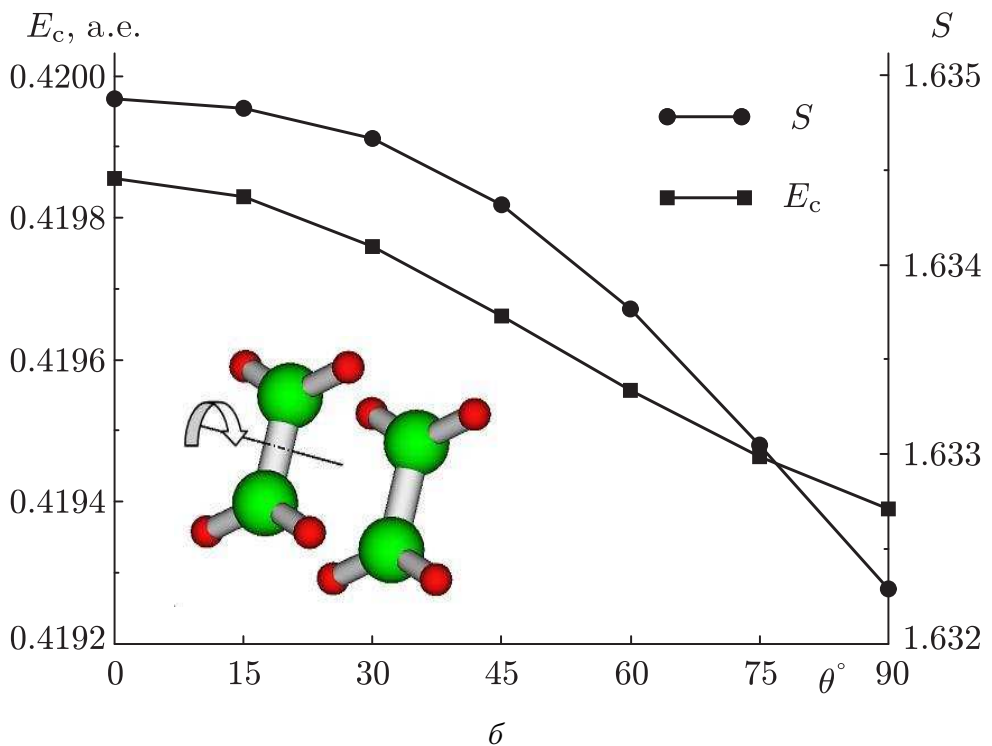

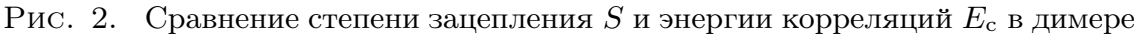
этилена, вычисленных при изменении взаимного расстояния между молекулами, взятыми в ориентации “лицом к лицу" (а), и при изменении взаимной ориентации, молекулы параллельны и находятся на расстоянии $R=3.5 \AA$ (б). 
ми, то эти две функции быстро убывают вплоть до расстояния $R=4.5 \AA$ А, а при бо́льших расстояниях начинают убывать медленнее. Если положить расстояние равным $R=3.5 \AA$ и изменить взаимную ориентацию молекул, оставив их в своих плоскостях, то как зацепление, так и энергия корреляции убывают с ростом угла поворота. Это связано с тем, что при вращении молекулы в ее плоскости чем больше угол поворота, тем меньше суперпозиция внешних орбиталей двух указанных молекул. Соответственно, зацепление электронов и энергия корреляции убывают, что и показано на рис. 2.

Из приведенных выше рассуждений следует, что зацепление можно использовать как эффективный инструмент при расчете энергий электронных корреляций не только в молекуле $\mathrm{H}_{2}$ [12], но и в более сложных системах.

\section{4. РЕЗУЛЬТАТЫ: ВЗАИМОДЕЙСТВУЮЩИЕ МОЛЕКУЛЫ}

Наши исследования степени зацепления электронов представляются полезными при изучении квантовых компьютеров, входные состояния которых подготавливаются так, чтобы эти состояния были максимально зацеплены. На этом пути представляется возможным добиться экспоненциального ускорения квантовых вычислений по сравнению с классическими. Главная задача данной работы состоит в анализе димера этилена, который представляет собой простейшую органическую связанную систему. Цель этого анализа - найти конфигурацию, отвечающую максимальной степени зацепленния между двумя молекулами. При этом мы рассматриваем составную систему как двудольную систему, в которой каждая из молекул может рассматриваться как кубит, и затем вычисляем степень зацепления, вызванного только взаимодействием между молекулами, пренебрегая взаимодействиями внутри каждой из молекул.

Для реализации нашей цели используем хорошо известный в квантовой химии факт: энергию корреляций между двумя молекулами этилена можно получить как разность между энергией корреляций, обусловленной взаимодействиями между всеми электронами в составной системе, и энергией корреляций электронов в каждой из молекул, т.е.

$$
E_{\mathrm{corr}}^{\mathrm{int}}=E_{\mathrm{c}}\left[2 \mathrm{C}_{2} \mathrm{H}_{4}\right]-2 E_{\mathrm{c}}\left[\mathrm{C}_{2} \mathrm{H}_{4}\right] .
$$

Если расстояние между двумя молекулами $\mathrm{C}_{2} \mathrm{H}_{4}$ бесконечно, то их можно рассматривать как две отдельные подсистемы составного димера, который мы обозначаем через $2 \mathrm{C}_{2} \mathrm{H}_{4}$, поскольку в этом случае подсистемы некоррелированы. Тогда $\rho\left[2 \mathrm{C}_{2} \mathrm{H}_{4}\right]=\rho\left[\mathrm{C}_{2} \mathrm{H}_{4}\right] \otimes \rho\left[\mathrm{C}_{2} \mathrm{H}_{4}\right]$. Применяя определение фон неймановской энтропии, получим, что $S\left(\rho\left[2 \mathrm{C}_{2} \mathrm{H}_{4}\right]\right)=2 S\left(\rho\left[\mathrm{C}_{2} \mathrm{H}_{4}\right]\right)$. В общем случае, т.е. при конечных расстояниях, имеет место неравенство Клейна

$$
S\left(\rho\left[2 \mathrm{C}_{2} \mathrm{H}_{4}\right]\right)>S\left(\rho\left[\mathrm{C}_{2} \mathrm{H}_{4}\right]\right)+S\left(\rho\left[\mathrm{C}_{2} \mathrm{H}_{4}\right]\right)=2 S\left(\rho\left[\mathrm{C}_{2} \mathrm{H}_{4}\right]\right) .
$$

Таким образом, мы определяем степень защепления взаимодействующих электронов как

$$
S_{\mathrm{int}}=S\left(\rho\left[2 \mathrm{C}_{2} \mathrm{H}_{4}\right]\right)-2 S\left(\rho\left[\mathrm{C}_{2} \mathrm{H}_{4}\right]\right)
$$


Исследуем теперь степень зацепления взаимодействующих электронов в димере этилена при изменении взаимной ориентации и расстояния между молекулами. Результаты расчета приведены на рис. 3. На этом рисунке степень зацепления взаимодействующих электронов представлена как функция от угла $\theta$ поворота при различных значениях расстояния $R$ между молекулярными плоскостями (рис. За, 3б) и как функция от межмолекулярного расстояния $R$ при различных углах поворота $\theta$ (рис. 3в), вычисленная в конфигурации “лицом к лицу". Видно, что при $R<3.1 \AA$ величина $S_{\text {int }}$ как функция угла поворота имеет колоколообразную форму, и ее значения тем больше, чем больше межмолекулярное расстояние. Для различных значений межмолекулярного расстояния максимум $S_{\text {int }}$ достигается при $30^{\circ} \leqslant \theta \leqslant 50^{\circ}$. Более того, чем ближе молекулы, тем на меньший угол надо их повернуть друг относительно друга, чтобы достичь максимального зацепления. Напротив, при $R>3.1 \AA$ величина $S_{\text {int }}$ убывает с ростом $R$ и в меньшей степени зависит от угла вращения, так как молекулы становятся все менее и менее коррелироваными. Чтобы подтвердить это поведение, мы представили $S_{\text {int }}$ как функцию от $R$ при различных углах пово-

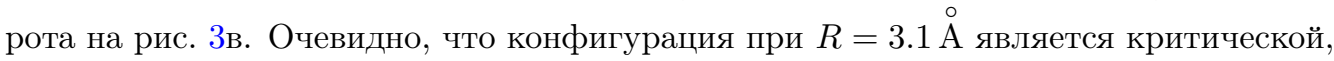
т.е. максимально зацепленной. В самом деле, при меньших расстояниях энтропия возрастает, в то время как при бо́льших расстояниях она убывает с ростом $R$. Кроме того, при $R=5.5 \AA$ А корреляция между двумя молекулами становится пренебрежимо малой при любых от углах поворота.

\section{5. ДЕТАЛИ ВЫЧИСЛЕНИЙ}

Для вычисления коэффициентов волновой функции в CISD-методе или, эквивалентно, элементов редуцированной матрицы плотности $\rho_{1}^{\mathrm{CISD}}$, а также для того чтобы найти собственные значения матрицы плотности, т.е. естественные спиновые орбитали, был использован пакет программ Gaussian 03 [29].

При расчете диссоциации молекулы $\mathrm{H}_{2}$ волновая функция раскладывалась с помощью CISD-метода. В этой системе возможны лишь одинарные или двойные возмущения, а потому CISD-метод оказывается методом полных конфигурационных взаимодействий (FCI-методом). При исследованиях димера этилена волновая функция раскладывалась с помощью метода связанных кластеров один-два (CCSD-метода) [30], [31]. Он является численным методом, используемым при описании многочастичных систем, и может быть применен для расчета корреляционных эффектов в системе двух связанных тел. CCSD-метод представляет собой обрезанный по взаимодействию метод связанных кластеров (СС-метод). Это означает, что используются только синглетные и дублетные операторы возмущений, следовательно, этот метод не может рассматриваться как подобный FCI-методу. Тем не менее, с его помощью можно описывать взаимодействие отдельных молекул лучше, чем в CISD-методе [23].

Для обеих систем (молекулы $H_{2}$ и димера этилена) входное состояние подготавливливалось с помощью вычислений по неограниченному методу Хартри-Фока 

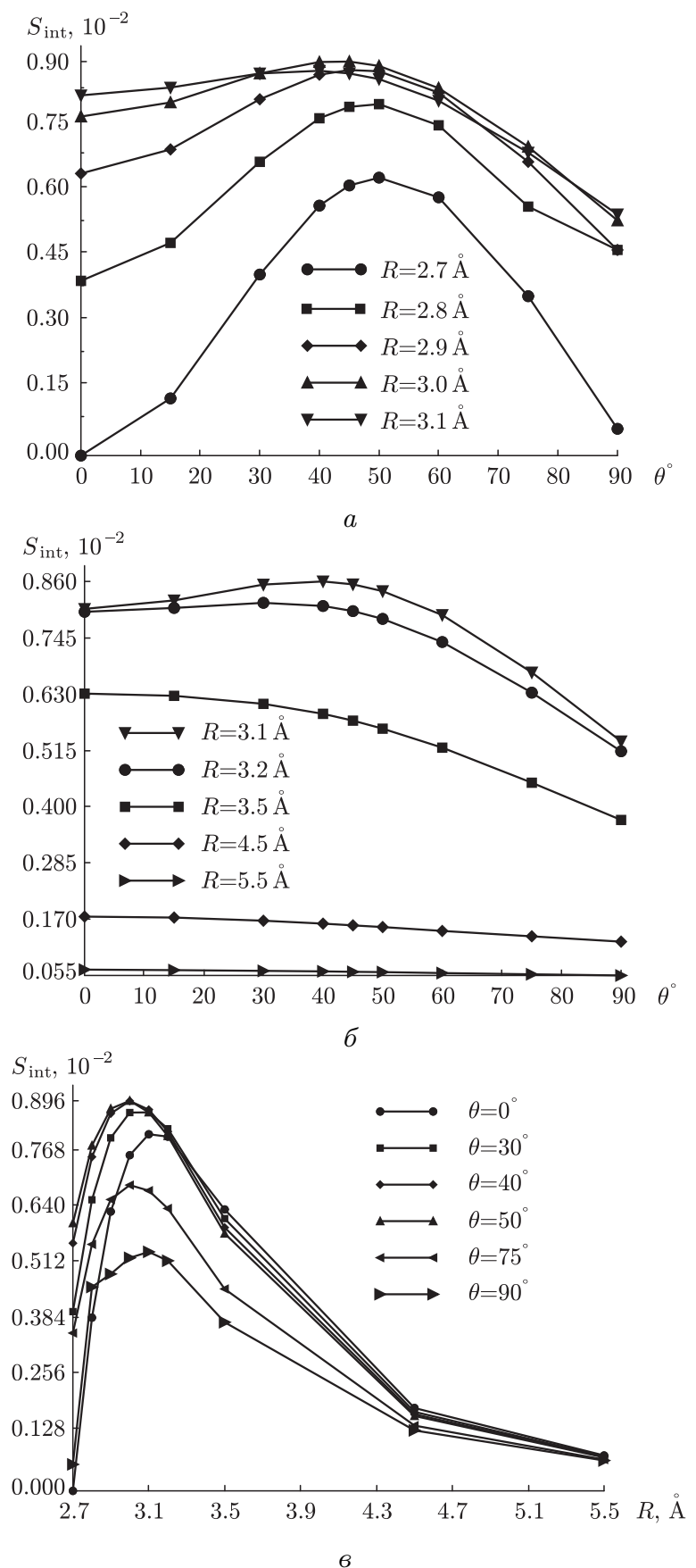

Рис. 3. Степень зацепления взаимодействующих электронов в димере этилена как функция от угла поворота, вычисленная при различных расстояниях $R$ между плоскостями молекул в конфигурации “лицом к лицу”(а, б). Та же величина как функция от межмолекулярного расстояния при различных углах поворота (в). 


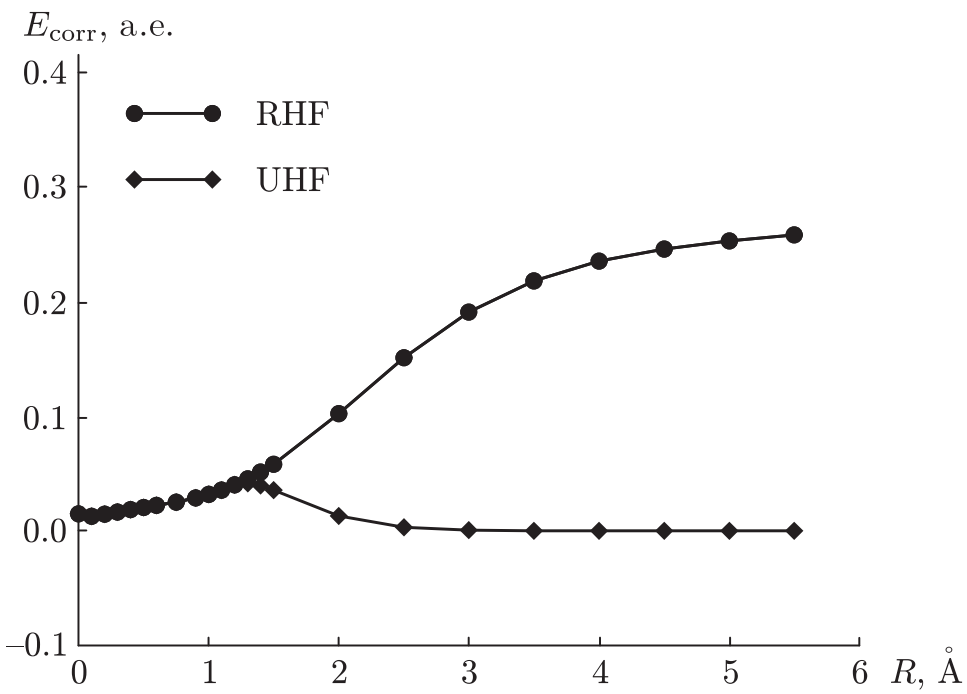

Рис. 4. Корреляция электронов как функция от межъядерного расстояния $R$ в молекуле $\mathrm{H}_{2}$, вычисленная с использованием гауссова базисного набора $3-21 G$ в пакете программ Gaussian. Для сравнения приведены кривые, рассчитанные с помощью RHF- и UHF-методов.

(UHF-методу). На самом деле, описание разрыва связи с помощью UHF-метода в молекуле $\mathrm{H}_{2}$ приводит к правильным продуктам диссоциации, в то время как описание молекулы $\mathrm{H}_{2}$ с помощью ограниченного метода Хартри-Фока (RHF-метода) приводит к нереалистичным результатам. Таким образом, для того чтобы исследовать диссоциацию молекулы $\mathrm{H}_{2}$, необходимо задавать энергию корреляции электронов в приближении UHF-метода. При коротких межъядерных расстояниях волновые функции в RHF- и UHF-методах совпадают, но при больших расстояниях вне области связи только в UHF-методе удается достичь правильного поведения энергии корреляции молекулы $\mathrm{H}_{2}$, которая должна обращаться в нуль, когда два атома далеки друг от друга, и электроны не интерферируют (см. рис. 4).

Энергия корреляции в уравнении (12) задается в терминах полного одноэлектронного базиса. На практике, однако, при вычислениях энергии корреляций приходится использовать неполный базис. Мы будем применять термин энергия корреляиий для обозначениия энергии, полученной из уравнения (12) в неполном одноэлектронном базисе.

Амплитуда энергии корреляций обычно возрастает с увеличением размерности базиса орбиталей, так как базис низкой размерности обычно не обладает гибкостью, необходимой для аккуратного воспроизведения эффектов корреляций. Для подтверждения этой теории проанализируем четыре различных вида базисных наборов, известных в литературе как $3-21 G, 6-31 G, 6-31 G^{* *}$ и $6-31+G^{* *}$, и 
покажем, что увеличение размерности базиса приводит к росту вклада в энергию корреляций.

В базисных наборах $3-21 G$ и $6-31 G$ участвуют атомные орбитали $1 s, 2 s$ и $1 p$, и потому их вклады в энергию корреляций примерно совпадают. Базисные наборы $6-31 G^{* *}$ и $6-31+G^{* *}$ включают в рассмотрение также орбиталь $1 d$. Энергия корреляции, полученная в последних двух базисах, превышает ту, которая была получена с помощью первых базисных наборов. Важно тем не менее отметить, что при малых межъядерных расстояниях энергия корреляции существенно зависит от размерности базиса орбиталей, в то время как при больших расстояниях вне области связи результаты использования всех четырех базисов оказываются примерно одинаковыми.

После этого предварительного исследования базисных наборов мы остановили свой выбор на использовании наименьшего базисного набора $3-21 G$. Это позволило радикально сократить время вычислений, что особенно привлекательно в связи с исследованием других систем, в частности, более сложных молекул.

\section{6. ЗАКЛЮЧЕНИЕ}

В данной работе мы проанализировали и конкретизировали гипотезу работы [12] о том, что зацепление может быть использовано в качестве альтернативной оценки корреляции электронов при вычислениях в области квантовой химии.

Сначала мы сравнили степень зацепления в процессе диссоциации молекулы $\mathrm{H}_{2}$, которую мы вычисляли как фон неймановскую энтропию редуцированной матрицы плотности $S\left(\rho_{1}\right)$ (как и в работе [12]), с аналогичной величиной, вычисленной с помощью энтропии матрицы плотности составной системы $S(\rho)$. Так как поведение обеих величин оказывается сходным, мы предположили, что $S(\rho)$ может служить качественной оценкой степени зацепления и в более сложных системах. Затем мы проверили, что энергия корреляции электронов в димере этилена хорошо воспроизводится с помощью степени зацепления $S(\rho)$, вычисленной для разнообразных конфигураций системы, показанных на рис. 2.

Производя анализ взаимодействующих молекул, мы ввели понятие зацепления взаимодействующих электронов, с помощью которого можно вычислить степень зацепления, обусловленного одним лишь межмолекулярным взаимодействием без учета внутренних корреляций молекул. Как показано на рис. 3, имеют место конфигурации, на которых степень зацепления достигает своего максимума. Анализ степени зацепления электронов оказывается полезным для исследования квантовых компьютеров, входные состояния которых конструируются так, чтобы они были максимально зацеплены.

\section{ПРИЛОЖЕНИЕ}

Покажем, как можно вычислить редуцированную матрицу плотности (9). Для упрощения вычислений рассмотрим случай двух электронов в двухуровневой систе- 
ме. Рассмотрим гильбертово пространство (1) при $m=2$ :

$$
\mathcal{H}=\left[\mathcal{L}^{2}(1) \otimes \mathcal{S}^{2}(1)\right] \otimes\left[\mathcal{L}^{2}(2) \otimes \mathcal{S}^{2}(2)\right]=\mathcal{C}^{4}(1) \otimes \mathcal{C}^{4}(2),
$$

где через $\mathcal{L}$ и $\mathcal{S}$ обозначены соответствующие орбитальное и спиновое пространства. В представлении чисел заполнения чистое двухэлектронное состояние $|\Psi\rangle$ можно представить в виде $|\Psi\rangle=\sum_{a=1}^{4} \sum_{b=1}^{4} \omega_{a, b} c_{a}^{\dagger} c_{b}^{\dagger}|0\rangle$, где $|0\rangle$ - вакуумное состояние, коэффициент $\omega$ и операторы рождения-уничтожения определены формулами (4) и (5) при надлежащем выборе $m$. Рассмотрим теперь четыре узла вместо двух частиц: они представляют собой узлы, которые либо не содержат частиц, либо содержат одну частицу. Так как нашей целью является получение информации о величине $n_{1}$, вычислим частичный след оператора плотности $\rho$ по состояниям $n_{2} \uparrow$ и $n_{2} \downarrow$ :

$$
\begin{aligned}
\rho_{n_{1}} & =\operatorname{Tr}_{n_{2}} \rho=\sum_{\substack{n_{2} \uparrow=0,1 \\
n_{2} \downarrow=0,1}}\left\langle n_{2} \uparrow, n_{2} \downarrow|\rho| n_{2} \uparrow, n_{2} \downarrow\right\rangle= \\
& =\langle 00|\rho| 00\rangle+\langle 01|\rho| 01\rangle+\langle 10|\rho| 10\rangle+\langle 11|\rho| 11\rangle .
\end{aligned}
$$

Найдем каждый из матричных элементов по отдельности.

Первый матричный элемент имеет вид

$$
\langle 00|\rho| 00\rangle=\sum_{a, b=1}^{4} \sum_{a^{\prime}, b^{\prime}=1}^{4} \omega_{a, b} \omega_{a^{\prime}, b^{\prime}}^{*}\left\langle 00\left|c_{a}^{\dagger} c_{b}^{\dagger}\right| 0\right\rangle\left\langle 0\left|c_{b^{\prime}} c_{a^{\prime}}\right| 00\right\rangle,
$$

где $a, b, a^{\prime}$ и $b^{\prime}$ принимают значения $1,2,3,4$ и представляют собой четыре узла исследуемой двухуровневой системы; более того, поскольку мы вычисляем внутреннее произведение в обкладках $\langle 00|$ и $|00\rangle$, единственным членом в сумме, дающим ненулевой вклад, будет $|1100\rangle^{1)}$, и ему соответствуют $\omega_{1,2}$ и $\omega_{2,1}$ :

$$
|1100\rangle: \quad\left|n_{1} \uparrow\right\rangle \otimes\left|n_{1} \downarrow\right\rangle \rightarrow \omega_{1,2}, \quad\left|n_{1} \downarrow\right\rangle \otimes\left|n_{1} \uparrow\right\rangle \rightarrow \omega_{2,1} .
$$

Тем самым

$$
\langle 00|\rho| 00\rangle=\sum_{a, b=1}^{2} \sum_{a^{\prime}, b^{\prime}=1}^{2} \omega_{a, b} \omega_{a^{\prime}, b^{\prime}}^{*}\left\langle 00\left|c_{a}^{\dagger} c_{b}^{\dagger}\right| 0\right\rangle\left\langle 0\left|c_{b^{\prime}} c_{a^{\prime}}\right| 00\right\rangle .
$$

Вычисляя суммы и используя условие $\omega_{i i}=0$, получим

$$
\begin{aligned}
\langle 00|\rho| 00\rangle=\omega_{1,2} \omega_{1,2}^{*}\left\langle 00\left|c_{1}^{\dagger} c_{2}^{\dagger}\right| 0\right\rangle\left\langle 0\left|c_{2} c_{1}\right| 00\right\rangle+\omega_{2,1} \omega_{1,2}^{*}\left\langle 00\left|c_{2}^{\dagger} c_{1}^{\dagger}\right| 0\right\rangle\left\langle 0\left|c_{2} c_{1}\right| 00\right\rangle+ \\
+\omega_{1,2} \omega_{2,1}^{*}\left\langle 00\left|c_{1}^{\dagger} c_{2}^{\dagger}\right| 0\right\rangle\left\langle 0\left|c_{1} c_{2}\right| 00\right\rangle+\omega_{2,1} \omega_{2,1}^{*}\left\langle 00\left|c_{2}^{\dagger} c_{1}^{\dagger}\right| 0\right\rangle\left\langle 0\left|c_{1} c_{2}\right| 00\right\rangle .
\end{aligned}
$$

Действие операторов рождения и уничтожения на вакуумное состояние порождает такие состояния, как $\pm\left|n_{1} \uparrow n_{1} \downarrow\right\rangle$ и $\pm\left|n_{1} \downarrow n_{1} \uparrow\right\rangle$, где выбор знака зависит от порядка действия операторов. Используя в дальнейшем представление, связанное с узлами,

1) Во всех этих вычислениях каждый четырехкомпонентный вектор имеет первую и вторую составляющие, отвечающие двум узлам уровня $n_{1}$, а третья и четвертая составляющие отвечают уровню $n_{2}$. Например, кет-вектор $|1100\rangle$ отвечает случаю, когда два электрона присутствуют на уровне $n_{1}$ и ни одного электрона не содержится на уровне $n_{2}$. 
а не с фермионами, мы можем представить все эти состояния как $\pm|1100\rangle$, при этом уравнение (П.1) примет вид

$$
\begin{aligned}
&\langle 00|\rho| 00\rangle=\omega_{1,2} \omega_{1,2}^{*}\langle 00 \mid 1100\rangle\langle 1100 \mid 00\rangle-\omega_{2,1} \omega_{1,2}^{*}\langle 00 \mid 1100\rangle\langle 1100 \mid 00\rangle- \\
&-\omega_{1,2} \omega_{2,1}^{*}\langle 00 \mid 1100\rangle\langle 1100 \mid 00\rangle+\omega_{2,1} \omega_{2,1}^{*}\langle 00 \mid 1100\rangle\langle 1100 \mid 00\rangle .
\end{aligned}
$$

Используя свойство ортогональности, получим, что $\langle 00|\rho| 00\rangle=4\left|\omega_{1,2}\right|^{2}|11\rangle\langle 11|$.

Проанализируем теперь второй матричный элемент:

$$
\langle 01|\rho| 01\rangle=\sum_{a, b=1}^{4} \sum_{a^{\prime}, b^{\prime}=1}^{4} \omega_{a, b} \omega_{a^{\prime}, b^{\prime}}^{*}\left\langle 01\left|c_{a}^{\dagger} c_{b}^{\dagger}\right| 0\right\rangle\left\langle 0\left|c_{b^{\prime}} c_{a^{\prime}}\right| 01\right\rangle .
$$

Так как внутреннее произведение берется в обкладках $\langle 01|$ и $|01\rangle$, то единственными элементами в сумме, дающими ненулевые вклады, будут $|1001\rangle$ и $|0101\rangle$, и они соответствуют $\omega_{1,4}, \omega_{4,1}, \omega_{2,4}$, и $\omega_{4,2}$ :

$$
\begin{aligned}
& |1001\rangle: \quad\left|n_{1} \uparrow\right\rangle \otimes\left|n_{2} \downarrow\right\rangle \rightarrow \omega_{1,4}, \quad\left|n_{2} \downarrow\right\rangle \otimes\left|n_{1} \uparrow\right\rangle \rightarrow \omega_{4,1} ; \\
& |0101\rangle: \quad\left|n_{1} \downarrow\right\rangle \otimes\left|n_{2} \downarrow\right\rangle \rightarrow \omega_{2,4}, \quad\left|n_{2} \downarrow\right\rangle \otimes\left|n_{1} \downarrow\right\rangle \rightarrow \omega_{4,2} .
\end{aligned}
$$

Тем самым

$$
\langle 01|\rho| 01\rangle=\sum_{a, b=1,2,4} \sum_{a^{\prime}, b^{\prime}=1,2,4} \omega_{a, b} \omega_{a^{\prime}, b^{\prime}}^{*}\left\langle 01\left|c_{a}^{\dagger} c_{b}^{\dagger}\right| 0\right\rangle\left\langle 0\left|c_{b^{\prime}} c_{a^{\prime}}\right| 01\right\rangle .
$$

Вычисляя эту сумму и применяя, как и раньше, представление узлов, мы можем записать этот матричный элемент в виде

$$
\langle 01|\rho| 01\rangle=4\left|\omega_{1,4}\right|^{2}|10\rangle\langle 10| .
$$

Теперь для третьего матричного элемента

$$
\langle 10|\rho| 10\rangle=\sum_{a, b=1}^{4} \sum_{a^{\prime}, b^{\prime}=1}^{4} \omega_{a, b} \omega_{a^{\prime}, b^{\prime}}^{*}\left\langle 10\left|c_{a}^{\dagger} c_{b}^{\dagger}\right| 0\right\rangle\left\langle 0\left|c_{b^{\prime}} c_{a^{\prime}}\right| 10\right\rangle
$$

заметим, что единственными векторами, приводящими к ненулевым вкладам в сумму после взятия внутреннего произведения в обкладках $\langle 10|$ и $|10\rangle$, будут

$$
\begin{aligned}
& |1010\rangle: \quad\left|n_{1} \uparrow\right\rangle \otimes\left|n_{2} \uparrow\right\rangle \rightarrow \omega_{1,3}, \quad\left|n_{2} \uparrow\right\rangle \otimes\left|n_{1} \uparrow\right\rangle \rightarrow \omega_{3,1}, \\
& |0110\rangle: \quad\left|n_{1} \downarrow\right\rangle \otimes\left|n_{2} \uparrow\right\rangle \rightarrow \omega_{2,3}, \quad\left|n_{2} \uparrow\right\rangle \otimes\left|n_{1} \downarrow\right\rangle \rightarrow \omega_{3,2} .
\end{aligned}
$$

Таким образом, $\langle 10|\rho| 10\rangle=4\left|\omega_{2,3}\right|^{2}|10\rangle\langle 10|$. Наконец, последний матричный элемент имеет вид $\langle 11|\rho| 11\rangle=4\left|\omega_{3,4}\right|^{2}|00\rangle\langle 00|$.

Итак, в базисе $\{|00\rangle ;|01\rangle ;|10\rangle ;|11\rangle\}$ матрица $\rho_{n_{1}}$ записывается как

$$
\rho_{n_{1}}=\left(\begin{array}{cccc}
4\left|\omega_{3,4}\right|^{2} & 0 & 0 & 0 \\
0 & 4\left|\omega_{2,3}\right|^{2} & 0 & 0 \\
0 & 0 & 4\left|\omega_{1,4}\right|^{2} & 0 \\
0 & 0 & 0 & 4\left|\omega_{1,2}\right|^{2}
\end{array}\right) .
$$


Это равенство задает оператор плотности на уровне $n_{1}$. Полезно обсудить его матричные элементы: $\omega_{3,4}$ представляет собой возмущения второго уровня, а потому в рассматриваемой двухуровневой системе величина $\omega_{3,4}$ связана с вероятностью того, что уровень $n_{1}$ оказывается пустым; величина $\omega_{1,2}$ связана с вероятностью того, что оба электрона находятся на уровне $n_{1}$, а коэффициенты $\omega_{2,3}$ и $\omega_{1,4}$ связаны с вероятностью того, что на уровне $n_{1}$ находится ровно один электрон. Это позволяет ввести следующие обозначения:

$$
\rho_{n_{1}, 0} \equiv 4\left|\omega_{3,4}\right|^{2}, \quad \rho_{n_{1}, 2} \equiv 4\left|\omega_{1,2}\right|^{2}, \quad \rho_{n_{1}, 1} \equiv\left(\begin{array}{cc}
4\left|\omega_{2,3}\right|^{2} & 0 \\
0 & 4\left|\omega_{1,4}\right|^{2}
\end{array}\right),
$$

где через $\rho_{n_{1}, 0}$ обозначена пустая орбиталь, через $\rho_{n_{1}, 2}-$ две заполненные орбитали, а через $\rho_{n_{1}, 1}$ - орбиталь, занятая одним электроном. Чтобы получить информацию об одном из двух электронов, возьмем частичный след от $\rho_{n_{1}}$ относительно одного из узлов на уровне $n_{1}\left(n_{1} \uparrow, n_{1} \downarrow\right)$ :

$$
\rho_{1}=\operatorname{Tr}_{n_{1} \uparrow} \rho_{n_{1}}=\sum_{n_{1} \uparrow=0,1} \rho_{n_{1}}=\left(\begin{array}{cc}
4\left(\left|\omega_{3,4}\right|^{2}+\left|\omega_{2,3}\right|^{2}\right) & 0 \\
0 & 4\left(\left|\omega_{1,4}\right|^{2}+\left|\omega_{1,2}\right|^{2}\right)
\end{array}\right) .
$$

В терминах коэффициентного разложения в CISD-методе амплитуды перехода $\omega_{i, j}$ можно записать как $\left|\omega_{1,2}\right|^{2}=c_{0}^{2} / 4,\left|\omega_{1,4}\right|^{2}=c_{2}^{2} / 4,\left|\omega_{3,4}\right|^{2}=c_{1,2}^{2} / 4$ и $\left|\omega_{2,3}\right|^{2}=c_{1}^{2} / 4$, поэтому матрица $\rho_{1}$ принимает вид

$$
\rho_{1}^{\mathrm{CISD}}=\left(\begin{array}{cc}
\left|c_{1}\right|^{2}+\left|c_{1,2}\right|^{2} & 0 \\
0 & \left|c_{0}\right|^{2}+\left|c_{2}\right|^{2}
\end{array}\right) .
$$

Уравнение (9) было получено с помощью обобщения этой модели на случай $m$-уровневой системы.

Благодарности. Работа частично поддержана Italian MUR (PRIN "Sintesi") и INFN (Project № LE41).

\section{Список литературы}

[1] M. A. Nielsen, I. L. Chuang, Quantum Computation and Quantum Information, Cambridge, Cambridge Univ. Press, 2000.

[2] C. H. Bennet, G. Brassard, C. Crépeau et al., Phys. Rev. Lett., 70 (1993), 1895.

[3] C. H. Bennet, S. J. Wiesner, Phys. Rev. Lett., 69 (1992), 2881.

[4] A. K. Ekert, Phys. Rev. Lett., 67 (1991), 661.

[5] C. A. Fuchs, Phys. Rev. Lett., 79 (1997), 1162.

[6] S. Ghosh, T. F. Rosenbaum, G. Aeppli, S. N. Coppersmith, Nature, 425 (2003), 48.

[7] Y. Chen, P. Zanardi, Z. D. Wang, F. C. Zhang, New J. Phys., 8 (2006), 97; quant-ph/0407228.

[8] L. He, G. Bester, A. Zunger, Phys. Rev. B, 72 (2005), 195307; cond-mat/0503492.

[9] F. Buscemi, P. Bordone, A. Bertoni, Phys. Rev. A, 73 (2006), 052312; quant-ph/0602127.

[10] J. Schliemann, J. I. Cirac, M. Kuś et al., Phys. Rev. A, 64 (2001), 022303.

[11] G. C. Ghirardi, L. Marinatto, Phys. Rev. A, 70 (2004), 012109.

[12] Z. Huang, S. Kais, Chem. Phys. Lett., 413 (2005), 1.

5 Теоретическая и математическая физика, т. 152, № 2, 2007 г. 
[13] W. Harneit, Phys. Rev. A, 65 (2002), 032322.

[14] D. M. Collin, Z. Naturforsch A, 48 (1993), 68.

[15] J. C. Ramírez, C. Soriano, R. O. Esquivel et al., Phys. Rev. A, 56 (1997), 4477.

[16] A. Szabo, N. S. Ostlund, Modern Quantum Chemistry. Introduction to Advanced Electronic Structure Theory, McGraw-Hill, New York, 1989.

[17] E. Schrödinger, Naturwissenschaften, 23 (1935), 807.

[18] A. C. Doherty, P. A. Parrilo, F. M. Spedalieri, Phys. Rev. Lett., 88 (2002), 187904.

[19] B. d'Espagnat, Conceptual Foundations of Quantum Mechanics, 2nd ed., Math. Phys. Monogr. Ser., 20, Benjamin, Reading, MA, 1976.

[20] И. фон Нейман, Математические основы квантовой механики, Наука, М., 1964.

[21] J. R. Gittings, A. J. Fisher, Phys. Rev. A, 66 (2002), 032305.

[22] J. Paldus, P. E. S. Wormer, F. Visser, A. van der Avoird, J. Chem. Phys., 76 (1982), 2458.

[23] G. E. Scuseria, A. C. Scheiner, T. J. Lee et al., J. Chem. Phys., 86 (1987), 2881.

[24] P. Zanardi, Phys. Rev. A, 65 (2002), 042101.

[25] C. H. Bennett, H. Bernstein, S. Popescu, B. Schumacher, Phys. Rev. A, 53 (1996), 2046.

[26] A. D. Gottlieb, N. J. Mauser, Phys. Rev. Lett., 95 (2005), 123003.

[27] R. Grobe, K. Rzazewski, J. H. Eberly, J. Phys. B, 27 (1994), L503.

[28] P. Gersdorf, W. John, J. P. Perdew, P. Ziesche, Internat. J. Quantum Chem., 61 (1997), 935.

[29] M. J. Frisch et al., Gaussian98, Revision A. 11.3, Gaussian, Pittsburgh, PA, 1998.

[30] J. Č́žzek, J. Chem. Phys., 45 (1966), 4256.

[31] G. E. Scuseria, C. L. Janssen, H. F. Schaefer III, J. Chem. Phys., 89 (1988), 7382. 